\title{
African One Health e-Surveillance Initiative
}

\author{
Joy Sylvester*1, Herbert Kazoora ${ }^{2}$, Meeyoung Park ${ }^{1}$, Sheba Gitta ${ }^{2}$, Betiel H. Haile ${ }^{1}$ and \\ Scott J. McNabb ${ }^{1}$
}

${ }^{1}$ Public Health Practice, LLC, Belmont, MA, USA; ${ }^{2}$ African Field Epidemiology Network, Kampala, Uganda

\section{Objective}

1) To establish One Health workgroups and conduct an e-Surveillance assessment to inform national strategic planning efforts in pilot countries. 2) To provide evidence for the African Surveillance Informatics Governance Board (ASIGB) to address its mission of establishing e-Surveillance.

\section{Introduction}

Information and Communication Technology (ICT) can enhance public health surveillance (PHS) by facilitating the digital exchange of information. Electronic surveillance (e-Surveillance) is the use of electronic systems to empower the digitization of PHS functions of prevention, detection, and response. E-Surveillance maximizes compliance with the International Health Regulations (2005), enables efficient Integrated Disease Surveillance and Response, and empowers One Health.

In Africa, e-Health is hindered by donor-funded, short-term projects known as "pilotitus." Proactive national leadership is required to establish a sustainable e-Surveillance program; an assessment and a strategic plan are the first steps.

Therefore, the One Health e-Surveillance Initiative (OHSI) was conceived and piloted by Public Health Practice, LLC (PHP) and the African Field Epidemiology Network (AFENET), with support by the Defense Threat Reduction Agency and the U.S. Centers for Disease Control and Prevention (CDC).

\section{Methods}

OHSI was piloted in Burkina Faso, Cameroon, Kenya, Nigeria, and Uganda from 2013 - 2015. One Health country-level work groups (CLWGs) were established and composed of medical epidemiologists, veterinary epidemiologists, laboratory scientists, informaticians, and clinicians. CLWG members were current employees of the Ministries of Health and Agriculture/Wildlife and were supported by the World Health Organization Regional Office for Africa country representatives. Their scopes of work included conducting a national e-Surveillance assessment and advocating for One Health e-Surveillance within their countries. PHP, AFENET, and CDC provided training and technical support for these efforts.

As part of the e-Surveillance assessment, CLWGs collected, cleaned, and analyzed data. They also interpreted data collected and wrote reports. Public health and veterinary surveillance units, health facilities, and laboratories at all administrative levels were assessed.

\section{Results}

OHSI stimulated formation of an African Surveillance Informatics Governance Board (ASIGB), chaired by the World Health Organization Regional Office for Africa (WHO/AFRO). OHSI established a framework consisting of the formation and training of One Health CLWG teams who conducted an e-Surveillance assessment. Additionally, a transnational e-Surveillance assessment tool and protocol capturing the variance of ICT capacity for e-Surveillance were developed. Owned by national Ministries, the assessment data collected will inform national strategic planning efforts. CLWG members are now advocates for One Health e-Surveillance.

\section{Conclusions}

ASIGB and CLWG creation, engagement, and ownership of the OHSI process and outcomes allowed for an in-depth understanding of the variance of ICT capacity to support e-Surveillance. The data collected through the assessment will support evidence-based strategic planning, and OHSI created champions of One Health e-Surveillance who can support this process.

Challenges included multiple languages, poor Internet connectivity, and time constraints of CLWG members due to the Ebola outbreak.

National strategic planning should occur using the assessment data collected. After incorporating feedback on the pilot processes and assessment, the ASIGB should support similar One Health e-Surveillance assessments in other African countries. OHSI should be replicated in other regions to support establishment of One Health e-Surveillance.

\section{Keywords}

e-Surveillance; One Health; Africa; Strategic planning; Evaluation

\section{Acknowledgments}

Ian Watson, Dylan Jones, Stephanie Fedrigo, Kenneth Ofosu-Barko, WHO/AFRO, Burkina Faso MoH, Burkina Faso MRA, Cameroon MINSANTE, Cameroon MINEPIA, Kenya MoH, Kenya MoA, Nigeria $\mathrm{FMoH}$, Nigeria FMARD, Uganda MoH, Uganda MAAIF, and CDC.

\author{
*Joy Sylvester \\ E-mail: jsylvester@publichealthpractice.com
}

